\title{
INCIDENCE OF COMMON TRANSFUSION TRANSMITTED DISEASES AMONG BLOOD DONORS
}

\author{
Ahmed MU ${ }^{1}$, Begum $\mathrm{HA}^{2}$, Hossain $\mathrm{T}^{3}$, Chakraborty $\mathrm{P}^{4}$
}

\begin{abstract}
Transfusion transmitted diseases are one of the major health problem in Bangladesh. This study was carried out among 12,270 voluntary and directed donors in the transfusion medicine department of Khulna Medical College Hospital (KMCH), a teaching hospital of southern Bangladesh during the period from 1st July 2007 to 30th June 2008 to see the incidence of HIV, HCV, HBV, Syphilis \& Malaria. All were voluntary and relative/directed donors. No professional donors had taken part in this donation system. Blood samples were collected in sterile dry plain test tube after predonation consent. Serum of each donor was collected by proper centrifugation within 6 hours of collection. Tests of all the samples were done within 24 hours of collection. The methods used were latex agglutination Immunochromatographic test (ICT) and reconfirmed by ELISA techniques. For malaria thick and thin film studies under microscope were done. On analyzing the results, $171(1.39 \%)$ were found positive for HBsAg, 03 $(0.024 \%)$ were positive for anti $\mathrm{HCV}, 01$ was positive for anti HIV $(0.008 \%)$. None found positive for Malarial parasite (MP) none were reactive for RPR test. Donation from professional blood donors are completely stopped in the transfusion medicine unit of KMCH. Mandatory screening of donated blood should be done for HIV, HBV, HCV, Syphilis and Malaria to reduce their transmission by transfusion.
\end{abstract}

Key Words: Transfusion transmitted diseases, Blood donors.

\section{Introduction}

Blood is life. There is no substitute for blood. Scientists agreed that artificial blood can not be manufactured by man in the foreseeable future ${ }^{1}$. Use of unscreened blood transfusion keep the patient at risk of acquiring many transfusion transmitted diseases (TTDs) like Hepatitis viruses (HBV, HCV), Human immune deficiency virus (HIV), Syphilis, Malaria etc. Unsafe sexual act is well known route for HBV, HCV, syphilis and HIV transmission. Since most of the routes for transmission prevail in this country, the spread of HBV, HCV, HIV, syphilis, malaria has become a great threat. Most of the donated blood is not screened properly for TTDs. Use of unscreened blood keeps the patient at risk of acquiring many TTDs.

Hepatitis-B virus infection results in broad spectrum of disease from sub-clinical infection to fulminate hepatitis. It can progress to chronic active hepatitis, cirrhosis of liver and hepatocellular carcinoma. Globally more than one million deaths occur from complication of $\mathrm{HBV}$ infection every year ${ }^{2}$. The most important laboratory test for the detection of early $\mathrm{HBV}$ infection is the immunoassay for HBsAg. HBsAg appears during the incubation period and detectable in most patients during the prodrome and acute stage. Its prolonged presence i.e. 6 months without symptom indicates the carrier state. A high rate of hepatocellular carcinoma occurs in chronic carriers $^{3}$.

Hepatitis C Virus (HCV) is emerging as one of the major health problem in Bangladesh. HCV is a spherical, enveloped RNA virus approximately $50 \mathrm{~mm}$ in diameter, which is yet to be clearly visualized under the electron microscope. HCV is mostly transmitted by transfusion of contaminated blood and blood products. Besides this, intravenous drug abuse, close personal contact, use of shared needle, razor etc. are also other subjected modes of transmission. About 3\% of the world's population is infected by this virus accounting a total of 170 million $\mathrm{HCV}$ infected persons globally ${ }^{4} \mathrm{HCV}$ infection is diagnosed by detecting antibodies to HCV by ELISA or by ICT. As false positive results can occur in the ELISA, a RIBA (recombinant immunoblot assay) should be performed as a confirmatory test.

HIV is one of the human retroviruses that preferentially infects and kills helper $\left(\mathrm{CD}_{4}\right) \mathrm{T}$ lymphocytes, resulting in the loss of cell mediated immunity and a high probability that the host will develop opportunistic infections ${ }^{5}$. HIV is not an endogenous virus of humans; i,e, no HIV sequences are found in normal human cell DNA. The origin of HIV and how it entered the human population remains uncertain. There is evidence that chimpanzees living in West Africa were the source of HIV-1. HIV-2 was isolated from AIDS patients in West Africa in 1986. The proteins of HIV-2 are only about $40 \%$ identical to those of the original HIV isolates. HIV-2 remains localized primarily to West Africa and is much less transmissible than HIV-I. The presumptive diagnosis of HIV infection is made by the detection of antibodies by

1. Dr. Musleh Uddin Ahmed, Associate Professor, Department of Transfusion Medicine, Comilla Medical College. 2. Dr. Hosne Ara Begum, Associate Professor, Department of Transfusion Medicine, Dhaka Medical College. 3. Dr. Tozammel Hossain, Medical Officer, Department of Transfusion Medicine, Khulna Medical College Hospital. 4. Professor. Prodipesh Chakraborty, Head, Department of Transfusion Medicine, Khulna Medical College Hospital. 
ELISA. Because there are some false positive results with this test, the definitive diagnosis is made by western blot analysis.

Treponema pallidum causes syphilis. T. pallidum is transmitted from spirochete containing lesions of skin or mucous membrane (eg, genitalia, mouth \& rectum) of an infected person to other persons by intimate contact. It can also be transmitted from pregnant women to their fetuses. ${ }^{6}$ Blood for transfusions collected during early syphilis is also infectious. The antigens of T. pallidum induce specific antibodies, which can be detected by immunoflurescence or haemagglutination tests in the clinical laboratory. They also induce nonspecific antibodies (reagin) which can be detected by the flocculation of lipids (cardiolipin) extracted from normal mammalian tissues e.g. beef heart. Flocculation tests, e.g. VDRL and RPR tests, detect the presence of these antibodies. These tests are positive in most cases of primary syphilis and are almost always positive in secondary syphilis. The titre of these nonspecific antibodies decrease with effective treatment, in contrast to the specific antibodies, which are positive for life.

Malaria is caused by four Plasmodia species of which $P$. vivax and $P$. falciparum are more common causes of malaria in Bangladesh. Worldwide malaria is one of the most common infectious diseases and a leading cause of death. Diagnosis rests on microscopic examination of blood, using both thick and thin Giemsa stained smears. The thick smear is used to screen for the presence of organism, and the thin smear is used for species identification. It is important to identify the species, because the disease process and treatment of different species may differ ${ }^{7}$.

\section{Materials and Methods}

This study was carried out among 12,270 voluntary and directed donors in the transfusion medicine department of Khulna Medical College Hospital (KMCH), a teaching hospital of Southern Bangladesh during the period from 1st July 2007 to 30th June 2008. The age distribution of

Table - I : Month wise screening report of five diseases.

\begin{tabular}{|c|c|c|c|c|c|c|c|c|c|c|c|c|c|c|}
\hline \multirow[b]{2}{*}{ Name of months } & \multicolumn{6}{|c|}{ Relative/Directed Donors } & \multicolumn{6}{|c|}{ Voluntary donors } & \multirow[b]{2}{*}{$\begin{array}{c}\text { Rejected } \\
\text { Donors }\end{array}$} & \multirow{2}{*}{$\begin{array}{c}\text { Total } \\
\text { Collected } \\
\text { Unit } \\
\end{array}$} \\
\hline & HIV & HBsAg & $\mathrm{HCV}$ & RPR & MP & Total & HIV & HBsAg & $\mathrm{HCV}$ & RPR & MP & Total & & \\
\hline July'07 & - & 21 & - & - & - & 1050 & - & - & - & - & - & 22 & 21 & 1072 \\
\hline August'07 & - & 11 & - & - & - & 1023 & - & - & - & - & - & 11 & 11 & 1034 \\
\hline September'07 & - & 12 & - & - & - & 1004 & - & - & - & - & - & 13 & 12 & 1017 \\
\hline October'07 & - & 21 & - & - & - & 963 & - & - & - & - & - & 11 & 21 & 974 \\
\hline November'07 & - & 15 & 1 & - & - & 1028 & - & - & - & - & - & 8 & 16 & 1036 \\
\hline December'07 & - & 14 & - & - & - & 923 & - & 1 & - & - & - & 24 & 15 & 947 \\
\hline January'08 & - & 20 & 1 & - & - & 919 & - & 1 & - & - & - & 8 & 22 & 927 \\
\hline February'08 & - & 15 & - & - & - & 1062 & - & - & - & - & - & 3 & 15 & 1065 \\
\hline March'08 & - & 13 & 1 & - & - & 1157 & - & - & - & - & - & 13 & 14 & 1170 \\
\hline April'08 & - & 9 & - & - & - & 1018 & - & - & - & - & - & 14 & 9 & 1032 \\
\hline May'08 & 1 & 10 & - & - & - & 958 & - & - & - & - & - & 6 & 11 & 964 \\
\hline June'08 & - & 8 & - & - & - & 1026 & - & - & - & - & - & 6 & 8 & 1032 \\
\hline Total & 1 & 169 & 3 & - & - & 12131 & - & 2 & - & - & - & 139 & 175 & 12270 \\
\hline
\end{tabular}

all the donors varied from 18 to 58 years. Blood samples were collected from voluntary and directed donors. It was not possible to cross examine the donors for clinical history such as history of jaundice, hepatitis, liver disease etc. that may provide clues for their infectiousness. A structured interview schedule was developed for collection of data. This was pretest on several blood donors for modification and finalization. Blood sample collected in the prelabeled pilot tube during collection of blood. Serum was separated from the clotted blood. The study included all the donors pretested blood grouping, $\mathrm{Rh}$ factor and cross matching with the blood of their respective recipient admitted in different wards of $\mathrm{KMCH}$. Detection of HBsAg, anti $\mathrm{HCV}$, and anti HIV were done by ICT. For screening of syphilis Rapid Precipitation Reaction (RPR) was carried out. Detection of malaria was done by making thick and thin film on the glass slides stained with Giemsa stain and Leishman stain respectively The serum of the donors blood were preserved in deep freezer for further laboratory tests of HBsAg, anti HCV,anti HIV by ELISA procedures. All tests were performed in accordance with the instruction of reagents manufacturer. Quality of the reagent was tested beforehand. Appropriate control was incorporated in all the test procedures. Data was collected based on the interview schedule through face to face interview with the respondent. The other necessary laboratory informations were collected after completion of the tests from the registar of transfusion medicine unit of $\mathrm{KMCH}$.

\section{Results}

The total number of blood samples tested was 12,270 of which 12,131 were directed donor and rest 139 samples were voluntary donor. Month wise screening picture of five diseases are shown in table-1. Possitivity rate of different transfusion tranmissible diseases is shown in the table-II.

\section{Discussion}

In the Blood Bank of Khulna Medical College Hospital not a single unit of blood of professional donor is entertained. But the percentage of voluntary donors was not so encouraging and was only $1.13 \%$. Prevalence of anti-HCV in the professional blood donors from the study done in the department of blood transfusion, Institute of Postgraduate Medicine and Research (IPGM \& R) in 1997 reported 5.5\% in apparently healthy persons $^{8}$. In the present study the sero prevalence 
Table - II : Positive cases among the donors of Transfusion Medicine Unit of KMCH. $(n=12270)$.

\begin{tabular}{|l|c|c|}
\hline \multicolumn{1}{|c|}{ Screening Tests } & Positive No. & Percentage \\
\hline Anti HIV & 1 & $0.008 \%$ \\
\hline HBV (HBsAg) & 171 & $1.39 \%$ \\
\hline Anti HCV & 3 & $0.024 \%$ \\
\hline VDRL (RPR) & 0 & Nil \\
\hline Malarial parasite & 0 & Nil \\
\hline
\end{tabular}

was only $0.024 \%$ which encouraged authority to promote voluntary blood donation.

Table - III shows the percentage of voluntary donors and directed (relative) donors in Khulna region. It is to be

Table - III : Relative frequency of voluntary donors and directed (relative) donors.

\begin{tabular}{|l|c|c|c|}
\hline Donors type & $\begin{array}{c}\text { No. of } \\
\text { donors }\end{array}$ & Percentage & $\begin{array}{c}\text { Total No. of } \\
\text { donors }\end{array}$ \\
\hline Directed (Relative) & 12131 & $98.87 \%$ & \multirow{2}{*}{12270} \\
\hline Voluntary & 139 & $1.13 \%$ & \\
\hline
\end{tabular}

noted here that during this study period there was no activities of blood donation of various social organizations (Sandhani, Medicine Club and Adhunik).

Blood donors in Bombay had $6 \%$ HBsAg positive. A study of prevalence of seromarkers in apparently healthy blood donors in Pakistan, HBsAg was positive in 5\% of the study group ${ }^{9}$. Serologic test results were obtained from multiple time volunteers at five USA blood centres from January 1991 through 1993 and it was found that the HBsAg incidence rate was 4.01 per $1,00,000$ person in a year $^{10}$. In this study it was about $1.39 \%$ but still it was statistically significant.

In this study, a single case of donor was found positive for anti HIV (0.008\%). Geographical difference may be a factor for low prevalence since all other studies were done in and around Dhaka. In this zone of Bangladesh, intravenous drug abusers and use of sharing needle is not so high, this may be considered as a co-factor. A study in the general population and professional blood donors may reveal the exact picture. The units with positive finding was confirmed by ELISA tests.It was safely discarded and the corresponding donors had gone through counseling for taking necessary measures against the concerned disease and were advised to remain aloof from further blood donation. RPR for $T$ pallidum and blood film stained with Giemsa stain for Malarial parasite (MP) were found absent in all donors but it does not mean that the total region is free from these diseases. Donation from professional donors is absolutely absent in the blood bank of $\mathrm{KMCH}$ so the picture of prevalence of diseases screened out are like this.

\section{Conclusion}

A study in general population including professional blood donors and commercial sex workers may reveal the exact picture. Mandatory screening of donated blood should be done for HIV, HCV, HBV, T. Pallidum, MP and other transfusion transmissible markers to ensure safe blood transfusion. Voluntary donor's blood is more safe so voluntary blood donation should be encouraged.

\section{Acknowledgement}

Thanks should be given to both medical technologist and other staff of the department of Transfusion Medicine, Khulna Medical College \& Hospital for their great help to carry out this study.

\section{Reference}

1. Rahman M. Guide to blood transfusion. 1st ed. Dhaka: Meghna Publication; 1978 .p.1-31.

2. Kane M. Primary hepatocellular carcinoma and cirrhosis of liver resulting from. Chr. Hepatitis-B virus infection. Vaccine 1995; 13:S47.

3. Levinson W, Jawetz E. Medical Microbiology \& Immunology. 7th ed. 2005.p. 260-262.

4. WHO Global surveillance and control of hepatitis-C. Report of a WHO consultation organized in collaboration with the viral hepatitis prevention board, Antwerp, Belgium. J Viral Hepat 1999; 6 : 35-47.

5. Population Report. Controlling sexually transmitted disease series, 1993; $9: 1-15$

6. Husain M, Islam MN, Sultan M, Prevalence of HBV, HCV, HIV \& syphilis markers in pregnant women in Bangladesh. Journal of BSMMU 1998; $13: 17$.

7. Das PC, et al. Transfusion Medicine 1996; $6: 319$ - 23.

8. Akbar MF, Hussain M, Hossain MF, Sarker S, Hossain SSS, et al, Seroepidemiology of Hepatitis virus of chronic liver disease in Bangladesh, high prevalence of HCV among Blood donors and healthy persons. Hepatology Research, Elsevier 1997; 113-120.

9. Rahman K, Khan AA, Haider Z, Syed SH, et al. Prevalence of seromarkers of HBV and HCV in health care personel and apparently healthy blood donors. J Pak Med Assoc 1996; 46 : 152-4

10. Kovelitz JJ, Bush MP, Schreiber GB, et al. A method for estimating Hepatitis B virus incidence rates in volunteer blood donors. Transfusion $1997 ; 37: 634-40$ 